\title{
A Reusable, Extensible Infrastructure for Augmented Field Trips
}

\author{
Mark J. Weal, Don Cruikshank, Danius T. Michaelides, \\ David E. Millard and David C. De Roure \\ IAM Group, University of Southampton, UK \\ $\{\mathrm{mjw}\}\{\mathrm{dgc}\}\{\mathrm{dtm}\}\{\mathrm{dem}\}\{\mathrm{dder}\} @$ ecs.soton.ac.uk \\ Eva Hornecker, John Halloran and Geraldine Fitzpatrick \\ Interact Lab, Dept. of Informatics, University of Sussex, UK \\ $\{$ eh49\}\{johnhall $\}\{$ G.A.Fitzpatrick\}@sussex.ac.uk
}

\begin{abstract}
This paper describes a reusable pervasive information infrastructure developed as part of the Equator IRC, designed to allow the construction of literacy based eLearning activities on top of material created as part of a more traditional visitors system.

The architecture of the system is described along with details of the creation of the curated material and the subsequent adaption of the system by local primary school teachers to create a literacy experiences. Results of the first trials of the system are presented with conclusions drawn and discussion of future directions.
\end{abstract}

\section{Introduction}

Wireless handheld technologies are increasingly being used in outdoor environments to provide engaging learning experiences for children in authentic settings. Much of the focus of this work to date had been about supporting scientific enquiry (Wireless Coyote [3], Gay et. al. [2], Ambient Wood [5]) and has often been set up as a one-off experience. The focus of this paper is to report on the first phase of a project that seeks to support a more diverse range of learning activities, initially creative writing, and to provide a persistent infrastructure that can easily be repurposed by teachers and others to create new experiences.

The Equator IRC (Interdisciplinary Research Collaboration) is a large UK project investigating the boundary between the physical and digital worlds. Chawton House Library is an Elizabethan manor house that once belonged to Jane Austen's brother Edward, and is now owned by a charitable foundation that operates it as a study centre of early English women's writing. A wide variety of visitors to the house can be identified including groups of schoolchildren using the grounds for a number of possible curriculum based experiences, academics studying at the Centre, coach parties from such as the Jane Austen Society of America, and local Flower Societies.

In order to move towards a generic infrastructure to support a wide range of visitors we have been working with the curators to produce a fairly standard visitors style system, and with teachers from Whiteley Primary School in Hampshire to create an augmented field trip for a group of Year 5 students (aged 10-11). An initial aim was to engage in a co-design process [4] with the curators at the house in order to create a basic visitors system where information can be relayed to the visitors at appropriate points in the grounds of the house. The focus of this paper is describing the infrastructure to support these experiences. The teachers from the school included the literacy advisor for the region. All the teachers are working closely with the relevant areas of the curriculum.

Other learning experiences for children using locative, context-based technologies have focused on scientific discovery, learning about nature, learning about history etc., Here, the main emphasis of the school experience has been on using the landscape as a writing aide. The children explored the grounds and constructed narratives around their discoveries. The teachers left instructions and activities for the children which flashed up on the PDAs at various locations around the grounds along with selected audio clips created by the curators as part of the separate visitors system. The pupils were asked to perform small tasks such as to record a dialogue between two people arriving at the house for the first time. The children can also record their own annotations for access later in the classroom 
when writing their stories.

The Chawton project architecture has been designed to support a wide range of different users and as a result a number of issues needed to be addressed. How can we enable the teachers and curators to create new experiences and engage in co-design? How can very different types of experiences be supported, i.e. experiences for different types of visitors to the house? How can we augment the grounds of a historic house without disturbing its atmosphere? How do we create an extensible and persistent infrastructure, that can be extended in terms of devices, infrastructure, content and types of experiences.

\section{The Card Metaphor}

To orchestrate the activities at Chawton we have applied a hypermedia technique called sculptural hypertext, coined by Mark Bernstein [1]. It describes the authoring of a hypertext system where initially every node is linked to every other node, and the authoring process removes links to produce the final hypergraph. These links are normally pruned using preconditions and assertions; in most systems describing themselves as sculptural, link pruning is dynamic. This differs from the more traditional calligraphic authoring such as hand authored Web pages, where new items are created unlinked and authors add links progressively to achieve the desired pattern of connection. This technique has been used in other pervasive projects to orchestrate the experience [6].

This system maintains a user context, or system state, and then decides which of the available cards held by the system fits with the current context. The user context includes user modelling of the participants as well as other environmental factors, in the case of the Chawton trials, the participants location as well as a global clock which controlled the overall experience. When a card is experienced by the participant, the user context can change as a result of the assertions held on the card. This allows us to build in prerequisites where a participant has to experience certain cards before they are able to access others. By encoding specific requirements on cards the experience designer gains control over the order in which events can happen as well as various timing requirements which might exist. This can be viewed as a form of state machine where the different context states are nodes in the hypermedia and the links between states are dynamically pruned based on the context information.

Different cards had different functionality associated with them, for instance a simple information card might have a piece of audio attached to it to be played automatically, or a capture card might ask the children to record a description of the particular part of the grounds they were in. Cards can then be arranged into sequences and decks for particular activities. The teachers were able to put specific constraints on the cards to ensure that an activity lasted a minimum amount of time or that the next card was played after a maximum allowed time for an activity.

By using a standardised ontology for representing the cards, the results of an activity (i.e. the recording of a description) can be made available to the other users of the system as information. In this way, the information cards created by the curators could be included in sequences in the literacy scenario.

\section{The Chawton Architecture}

Figure 1 shows an overview of the Chawton architecture. The pervasive infrastructure comprised at the base level: wireless networking, location sensing technology (GPS and RF pingers) as well as PDAs carried by the children.

On top of this infrastructure sat the generic information system, an orchestration tool (constructed using EQUIP, the EQUator Infrastructure Platform) alongside a triple store (the AKT 3store). The AKT 3Store is a MySQL based triple store that provides the facility to store and query RDF statements. It stored the cards used by the system during the trials. This was responsible for delivering information to participants, either visitors using the visit system or children taking part in the literacy experience. Orchestration was based on participants current context and metadata attached to the content.

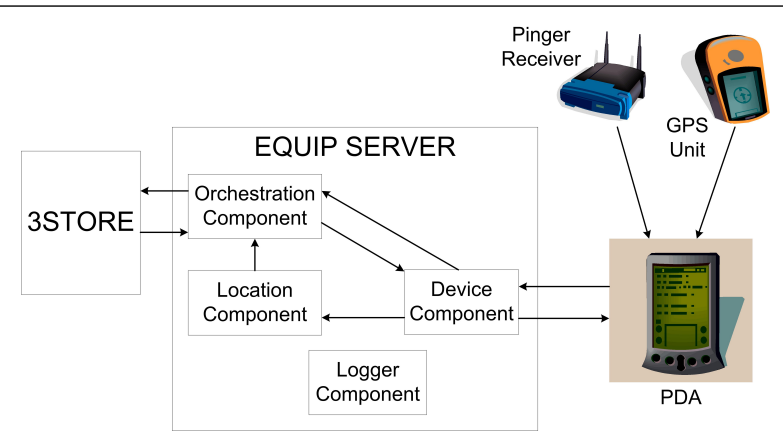

Figure 1. The Chawton project architecture.

The PDAs used in the trial were standard iPaq pocketPCs which communicated with a central server via wireless using HTTP. Four battery powered wireless access points were placed around the grounds to pro- 
vide coverage. Interaction with the PDA was through a single application which displayed the various cards and allowed the participants to listen to and record audio fragments, read textual instructions and make brief notes. The PDA sent information to the server and returned an XML document containing instructions for the software based on the matched context.

Location tracking was carried out using a combination of RF pingers and GPS. Each of the PDAs had an $\mathrm{RF}$ pinger receiver attached via the serial port which received pings from discrete pinger boxes placed around the grounds. The pingers, with a range of around 5 meters, were developed by the University of Bristol in a previous Equator project. This provided a mechanism for identifying and highlighting specific objects or locations such as the South Door or a specific tree that had been struck by lightning.

GPS was used for larger expanses, such as the lawn area or the walled gardens. GPS information was collected from a GPS receiver and sent via Bluetooth to the PDA. Pinger locations were given priority over GPS locations by the system in the event of both providing matches. The PDA did no processing of location information but simply forwarded it to the central server.

The architecture was constructed around the EQUIP framework ${ }^{1}$. This provides a component based tuple space designed for the development of the types of pervasive experiences we are interested in. The dataspace holds the current state of the system. As that state changes the components are informed of the changes in state they are interested in. The dataspace operates on a broadcast model but for simplicity the diagram indicates the flow of data around the components using arrows. The four main components that comprise the EQUIP system are:

Device Component: Containing the HTTP server used to communicate to and from the hand held devices. This processes information from the device into the tuple space then returns the current available deck for the device as an XML document.

Location Component: Parses the GPS and pinger information placed in the EQUIP tuple space. Where the information matches known locations it enters the symbolic location for the device into the tuple space.

Orchestration Component: Performs a query of the cards in the 3Store based on the current context (comprised of the device, deck

1 http://www.crg.cs.nott.ac.uk/ cmg/ Equator/Downloads/docs/equip-tech.pdf being used for the experience, current location, and previously viewed content). The result is turned into a deck which is placed into the tuple space.

Logger Component: Logs all traffic through the tuple space.

\section{The Curated Material}

The curators generated content both for their own experiences (tours) but also for re-use in other experiences such as the literacy scenario. For the initial trials, content creation involved the in-situ recording of audio content by the curators as they walked around the grounds. A post processing phase was required in order to segment the audio into individual clips and ppropriate context metadata was added. The intention is to produce a system that allows for ready revision and updating of the information content rather than aimed at providing a highly polished specific tour of the grounds.

Locations were defined in two ways. Either by the placement of a pinger used to indicate a region defined by being within range of the pinger or by walking out a region using the hand held device. A static deck of cards was produced which included paired instructions such as 'Go to the walled garden and press OK', followed by the instruction 'Walk slowly around the perimeter of the walled garden and press OK when completed.' The resultant system logs could then be automatically processed into GPS regions that defined the particular location.

\section{The Literacy Scenario}

The literacy scenario applied the infrastructure framework to the creation of a field trip for a group of children with the aim of steering them through the gathering of material for them to later use when writing a fictional story. The teachers devised a scenario where at various locations around the grounds the children would be given a range of different activities to carry out. Some required them to write in their exercise books while others involved the recording of text and audio on the PDAs.

The teachers were able to make use of most of the locations previously identified by the curators but were able to add some specific locations that were appropriate to the literacy exercise but had not featured in the material created previously. As well as creating the activities the teachers also selected information created by the curators that could be given to the children to augment their understanding of the landscape and house. It is this form of re-use that was one of the 
main design goals of the infrastructure developed for this project.

The teachers composed the literacy scenarios as sequences of activities to take place at named locations. These activities had various specific properties to do with the timing of the activity, the method of interaction used by the children and the order in which they take place. The created scenarios were converted into a deck of cards of the following types:

Information cards were selected from the information cards created by the curators and gave the children additional information about the various locations, perhaps after they'd been asked to think what it might have been used for.

Activity cards were designed to prompt the children to think more deeply about their surroundings. They might be ask to make notes in their paper notebooks, reflect on what might have occurred at a particular location or think about how different characters might react in the current place.

Capture cards required the children to record information digitally. This could be a piece of audio, some dialogue between two imaginary characters perhaps, or perahps a short piece of text. The children could then review or rerecord the information if they were unhappy with it.

Sequence cards were used to group a set of tasks together in a location. A simple sequence might be that the children listened to an audio clip about the stable block (Information), are asked to think about who might have worked there (Activity) and then finally record a short piece of dialogue taking place between their imagined characters (Capture).

As is true of most field trips, controlling the timing of the various activities is important. By modelling orchestration information as part of the ontology the teachers were able to specify how long the children should spend on particular activities and keep the overall activity progressing.

The logging of activities is all carried out using the same ontology allowing for easy reuse of the data collected during analysis post trials and also facilitating the re-use of information during the trials. It is hoped that the information space will evolve over time with the curated information increasing and experience builders such as the teachers being able to make use of cards from previous activities.

On returning to the school the children wrote their stories based on material they had collected at the house. An electronic journal of their activities was provided via the Web which allowed them to review their experience by seeing the deck of cards that they collected during their exploration. This allowed them to relisten to audio clips and access their own recordings and text.

\section{Initial Trials}

A group of children from Whiteley Primary School were brought out to Chawton House for a day of trials. They were given a guided tour of the insides of the house to set the scene before being divided into pairs for the later activities. Previously, at the school, they had been given a brief tutorial on how to use the PDA device. The literacy experience had two parts to it. In the first session the children explored the grounds freely and at various locations were required to carry out a sequence of activities. Figure 2 shows the children exploring in pairs.
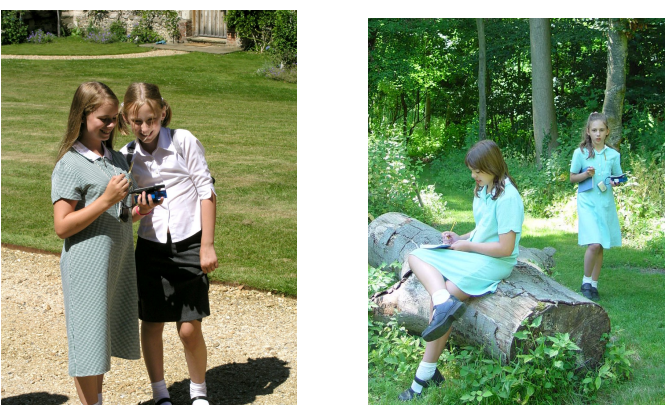

Figure 2. The device in use during the trial.

In the first phase the children gathered information through listening to audio prepared by the curators, following instructions set out by the teachers and observing the landscape, flora and fauna around them. Having completed the first phase the children were gathered together to briefly share their findings with each other before moving on to the second phase where they went back to two locations of their choice and received further instructions on more specific activities to help in the creation of their stories.

\subsection{Observations}

Both the children and teachers felt that the day had been a success and met their expectations. The head teacher commented on how highly motivated the children had been by the approach. The children were able, through the technology, to bring their experience back to the classroom, as one teacher put it, 'They all grabbed little bits of Chawton House yesterday. 
A problem encountered by teachers on more traditional field trips with clipboards is that the children often see the activities as a race in which the first to complete all the tasks wins. The ability to pace the activities by forcing the children to stop and reflect and not allowing them access to the next task until a fixed period of time had passed proved highly effective.

The children were readily accepting of the technology and quick to highlight what they liked about it during interviews. They enjoyed the freedom of being able to explore the various locations without having to stay to a fixed route. Comments made included that they preferred it to clipboards where the paper can blow around and become awkward to handle, they appreciated the authority of the voices speaking to them and enjoyed the fact they could relisten to information if they chose to. The children showed an awareness of some of the problems with the prototype system, namely speed of reaction and occasional software errors, but this did not seem to distract them and they were positive about the experience.

One of the main technical problems encountered has been the speed of response to changes in location. The asynchronous nature of the architecture may have exacerbated this along with the sheer speed of the children during the trials. As well as improving the speed of response, more directed tasks can be used to steer the participants to specific locations if required.

The encoding of the experience was in this instance a two phase process, with discussion with the teachers leading to a scenario document that was then created within the infrastructure by the researchers. This process has helped inform the requirements of a more automatic authoring system which will allow the teachers to perform this task themselves and remove the need for direct intervention.

As we had anticipated, this first trial has helped to give the curators and teachers a clearer idea of what we were working towards. Having seen the technology in action and understood more fully what it is capable of discussions about future trials have proved to be enthusiastic and highly productive.

\section{Conclusions}

The first trial with the Chawton prototype has allowed us to test the infrastructure and begin to investigate the issues raised when teachers create field trips using information provided by the curators of the house. We have focussed on a literacy experience for the children drawing on the usage of the house itself and the current curriculum for the group of children selected although the system is designed to be generic.
The use of sculptural hypermedia for orchestration provides a mechanism for encoding the complex interactions and dependencies and, with an appropriate context model, has been used to facilitate a rich experience encompassing a variety of content and interaction models. By using semantic web technology as the mediating layer between the presented interfaces (authoring and user experiences) and the underlying infrastructure, a unified approach can be taken that lends itself to ready reuse of material and the growing of a corpus of information.

For the next phase of the project we are developing tools to enable the curators and teachers to create their material more directly, and where possible in-situ. This will allow for the addition of richer material and the creation of a wider range of activities to stretch the infrastructure in different directions. We are planning further trials in the near future with both the curators and teachers as we begin to develop more advanced authoring tools. These will support the easy creation of re-usable content and augmented field trips moving towards a generic infrastructure that supports a wide range of activities.

\section{Acknowledgments}

The authors wish to acknowledge their partners: Chawton House Library, Whiteley Primary School and the University of Bristol. EPSRC IRC project "EQUATOR" GR/N15986/01.

\section{References}

[1] M. Bernstein. Card shark and thespis: exotic tools for hypertext narrative. In Proceedings of Hypertext '01, pages 41-50. ACM Press, 2001.

[2] G. Gay, R. Reiger, and T. Bennington. Using mobile computing to enhance field study. In Carrying the conversation forward, pages 507-528. Erlbaum, 2002.

[3] W. Grant. Wireless coyote: A computer-supported field trip. Comms ACM, 36(2):57-59, Feb. 1993.

[4] J. Halloran, E. Hornecker, G. Fitzpatrick, D. E. Millard, and M. J. Weal. The chawton house experience - augmenting the grounds of a historic manor house. In Workshop - Re-Thinking Technology in Museums: Towards a New Understanding of People's Experience in Museums, Limerick (Ireland), pages 54-65, June 2005.

[5] Y. Rogers, S. Price, D. S. Fraser, C. Randell, M. J. Weal, and G. Fitzpatrick. Ubi-learning Integrates indoor and outdoor experiences. Communications of the ACM, 48(1):55-59, Jan. 2005.

[6] M. J. Weal, D. T. Michaelides, M. K. Thompson, and D. C. De Roure. Hypermedia in the ambient wood. New Review of Hypermedia and Multimedia, Special Issue on Hypermedia beyond the Desktop, (9):137-156, 2003. 